\title{
A single dose of dexamethasone encapsulated in polyethylene glycol-coated polylactic acid nanoparticles attenuates cisplatin-induced hearing loss following round window membrane administration
}

\author{
Changling Sun ${ }^{1,3, *}$ \\ Xueling Wang ${ }^{1, *}$ \\ Zhaozhu Zheng² \\ Dongye Chen' \\ Xiaoqin Wang ${ }^{2}$ \\ Fuxin Shi' \\ Dehong $\mathrm{Yu}^{\prime}$ \\ Hao Wu'
}

'Department of OtolaryngologyHead and Neck Surgery, Xinhua Hospital, Ear Institute, Shanghai Jiao Tong University School of Medicine, Shanghai Key Laboratory of Translational Medicine on Ear and Nose Diseases, Shanghai, ${ }^{2}$ National Engineering Laboratory for Modern Silk, Soochow University, Suzhou, ${ }^{3}$ Department of Otolaryngology-Head and Neck Surgery, Affiliated Hospital of Jiangnan University, The Fourth People's Hospital of Wuxi City, Wuxi, People's Republic of China

*These authors have contributed equally to this work

Correspondence: Hao Wu Room 915, Science and Education Building, Kong Jiang Rd I665, Shanghai, People's Republic of China, 200092

Tel +86 2l 25078899

Fax +86 2I 65I5 6489

Email wuhao622@shI63.net

Dehong Yu

Room 9|4, Science and Education

Building, Kong Jiang Rd 1665, Shanghai,

People's Republic of China, 200092

Tel +86 2l 25078896

Fax +86 2I 65I5 6489

Email dehongyu@।26.com
This article was published in the following Dove Press journal:

International Journal of Nanomedicine

14 May 2015

Number of times this article has been viewed

Abstract: This study aimed to investigate the sustained drug release properties and hearing protection effect of polyethylene glycol-coated polylactic acid (PEG-PLA) stealth nanoparticles loaded with dexamethasone (DEX). DEX was fabricated into PEG-PLA nanoparticles using an emulsion and evaporation technique, as previously reported. The DEX-loaded PEG-PLA nanoparticles (DEX-NPs) had a hydrodynamic diameter of $130 \pm 4.78 \mathrm{~nm}$, and a zeta potential of $-26.13 \pm 3.28 \mathrm{mV}$. The in vitro release of DEX from DEX-NPs lasted 24 days in phosphate buffered saline ( $\mathrm{pH} 7.4$ ), 5 days in artificial perilymph (pH 7.4), and 1 day in rat plasma. Coumarin 6-labeled NPs placed onto the round window membrane (RWM) of guinea pigs penetrated RWM quickly and accumulated to the organs of Corti, stria vascularis, and spiral ganglion cells after 1 hour of administration. The DEX-NPs locally applied onto the RWM of guinea pigs by a single-dose administration continuously released DEX in 48 hours, which was significantly longer than the free DEX that was cleared out within 12 hours after administration at the same dose. Further functional studies showed that locally administrated single-dose DEX-NPs effectively preserved outer hair cells in guinea pigs after cisplatin insult and thus significantly attenuated hearing loss at $4 \mathrm{kHz}$ and $8 \mathrm{kHz}$ frequencies when compared to the control of free DEX formulation. Histological analyses indicated that the administration of DEX-NPs did not induce local inflammatory responses. Therefore, prolonged delivery of DEX by PEG-PLA nanoparticles through local RWM diffusion (administration) significantly protected the hair cells and auditory function in guinea pigs from cisplatin toxicity, as determined at both histological and functional levels, suggesting the potential therapeutic benefits in clinical applications.

Keywords: stealth nanoparticles, dexamethasone, single-dose administration, cisplatin-induced hearing loss

\section{Introduction}

Cisplatin (cis-diamminedichloroplatinum(II) [CDDP]) is a potent chemotherapeutic drug with an activity against a wide spectrum of cancers, especially squamous cell cancer of the head and neck region, in both pediatric and adult groups. ${ }^{1}$ The major dose-related adverse side effect of cisplatin treatment is irreversible sensorineural hearing loss. The reported rate of cisplatin-induced hearing loss ranges between $11 \%$ and $97 \%$, with an average incidence of $62 \% .^{2-4}$ Additionally, cisplatin-induced ototoxicity is more prevalent in children, affecting up to $90 \%$ of pediatric patients. ${ }^{5}$ Hearing loss is a common cause for depression and reduction in quality of life, ${ }^{6}$ and thus prevention 
and restoration of hearing loss induced by cisplatin is crucial for cancer patients, particularly young children.

The molecular mechanisms of cisplatin-induced hearing loss involve generation of reactive oxygen species (ROS) and depletion of the antioxidant glutathione. ${ }^{7}$ Specifically, cisplatin induces depletion of glutathione and antioxidant enzymes, and generates excess ROS. An increase of malondialdehyde levels subsequently promotes the influx of calcium into the cochlear cells and triggers apoptosis. ${ }^{1}$ Various researchers have attempted to develop effective otoprotective methods via the administration of antioxidants against ROS at an early stage in the ototoxic pathway. Unfortunately, many of the otoprotective agents inhibit the tumoricidal effects of cisplatin and/or have toxicities or unknown effects. ${ }^{8}$ No routine treatment modalities are currently available to act against cisplatin-induced hearing loss without affecting its chemotherapeutic activity in the clinic.

Glucocorticoids (prednisone, dexamethasone [DEX], methylprednisolone, etc) have been evaluated as potential otoprotective drugs based on their anti-inflammatory effects. ${ }^{9}$ However, systemic drug delivery of glucocorticoids is limited by the blood-cochlea barrier that restricts drugs from leaving the circulation and gaining access to the cells of the inner ear, resulting in no functional otoprotective activity against cisplatin-induced hearing loss. ${ }^{10}$ Furthermore, the systemically administered glucocorticoids can diminish the tumoricidal activity of cisplatin via downregulating apoptotic genes in tumor cells. ${ }^{11,12}$

Intratympanic drug delivery, allowing diffusion through the round window membrane (RWM) into the inner ear, is a common and routine strategy for the management of inner ear disease, such as sudden sensorineural hearing loss and Meniere's disease. ${ }^{13}$ This strategy avoids the "off-target" side effects associated with systemic delivery, improves the concentration of the drug delivered to the inner ear fluids, and reduces inhibitory effects on the chemotherapeutic activity of cisplatin. ${ }^{12}$ Recently, several studies have demonstrated that the intratympanic delivery of DEX minimized cisplatin-induced hearing in a frequency-related manner. ${ }^{14-17}$ The efficiency of this strategy in treating cisplatin-induced ototoxicity was further confirmed clinically. ${ }^{7}$ To date, the results obtained for the intratympanic delivery of glucocorticoids varied, highly depending on the dose, frequency, intensity of the cisplatin injection, and the animal models used in the experiments. ${ }^{18}$ In addition, frequent daily drug administration is required considering the loss of the drug down the Eustachian tube. The high frequency and high dose administration of intratympanic drugs would not only increase the cost of therapy, but also increase the incidence of tympanic membrane perforations ${ }^{19}$ and cause undesired infection during the procedure. ${ }^{20}$

In the present study, we investigated the therapeutic strategy of using polyethylene glycol-coated polylactic acid (PEG-PLA) stealth nanoparticles (NPs) to deliver DEX to the cochleae via penetration through the RWM and the resulting hearing protective potential against cisplatininduced hearing loss. A NP-based inner ear drug delivery system has emerged as a promising new avenue for delivering compounds to the cochlea in a sustained and controllable manner. The route of administration onto RWM has been established as an effective and minimally invasive approach to deliver drugs to the cochlea. ${ }^{21}$ Drug molecules, especially bioactive molecules, are usually functionally encapsulated in NPs, so that drug concentrations can be maintained at desired levels at the target sites for a long period of time, owing to the restricted diffusion of drug molecules and the controlled degradation of NPs. Previously, our group developed a well-defined, nontoxic PEG-PLA stealth NP drug delivery system for chemotherapeutic agents. ${ }^{22}$ Accordingly, we speculated that DEX-loaded PEG-PLA NPs (DEX-NPs) should facilitate sustained release of the drug, leading to a protection against cisplatin-induced hearing loss.

\section{Materials and methods Materials and animals}

Methoxy PEG-PLA (mPEG-PLA) (molecular weight [MW]: $50 \mathrm{kDa}$ ) was obtained from Daigang Biomaterial Co, Ltd (Ji'nan, People's Republic of China). DEX, purchased from Bristol-Myers Squibb (New York, NY, USA), was watersoluble. The DEX solution, referred to as DEX or free DEX, was dissolved in phosphate buffered saline (PBS) solution at $10 \mathrm{mg} / \mathrm{mL}$. Coumarin 6 was obtained from Sigma-Aldrich Co (St Louis, MO, USA). Rhodamine phalloidin and 4'6diamidino-2-phenylindole (DAPI) were purchased from Thermo Fisher Scientific (Waltham, MA, USA). All other chemicals were of analytical grade and used without further purification.

Male guinea pigs (4-6 weeks, weighing about $250 \mathrm{~g}$; number $[\mathrm{N}]=123$ ) were purchased from the Shanghai Laboratory Animal Center (Shanghai, People's Republic of China). All animals had free access to standard rat chow and water. The outline of the animal experiments was shown in Figure 1. The care and handling of animals were performed with the approval of the Institutional Authority for Laboratory Animal Care of Shanghai Jiao Tong University School of Medicine (Shanghai, People's Republic of China). 
The animal experiments designed in this study were approved by the Ethical Committee of Shanghai Jiao Tong University School of Medicine.

\section{Methods}

Preparation and characterization of DEX-loaded nanoparticles (DEX-NPs)

Preparation of DEX-NPs

DEX-NPs were fabricated using an emulsion and solvent evaporation method as described previously. ${ }^{22}$ Briefly, a mixture of mPEG-PLA and $\operatorname{DEX}(6 / 1, w / w)$ was dissolved in $1 \mathrm{~mL}$ of dichloromethane solution. Next, $3 \mathrm{~mL}$ of sodium cholate solution $(1 \%, \mathrm{w} / \mathrm{v})$ was slowly poured into the solution and sonicated at $320 \mathrm{w}$ for 30 seconds (Scientz Biotechnology Co, Ltd, Ningbo City, People's Republic of China). The resulting oil/water emulsion was further diluted in $36 \mathrm{~mL}$ of $0.5 \%$ sodium cholate solution and the organic solvent was removed via rotary evaporation under reduced pressure. The resulting NPs were collected by centrifugation $(11,000 \times g, 30$ minutes $)$ and washed twice to remove excess emulsifier. The NPs were frozen and stored at $-20^{\circ} \mathrm{C}$ for further use. Coumarin 6-labeled NPs were prepared using the same method as DEX-NPs, except that DEX was replaced with $0.05 \%(\mathrm{w} / \mathrm{v})$ Coumarin 6 .

\section{Morphology and particle size}

Particle size, as well as size distribution and morphology, were determined with three different methods: dynamic light scattering (DLS); transmission electron microscopy (TEM); and atomic force microscopy (AFM). DEX-NPs were diluted in double-distilled water and measured by DLS. DEX-NPs were negatively stained with sodium phosphotungstate solution and scanned with TEM. One drop of the NP suspension was mounted on metal slabs, air-dried, and scanned by the AFM with a Nanoscope III in the tapping mode. The zeta potential was measured using DLS.

Encapsulation efficiency (EE \%) and drug loading (DL \%) of the DEX-NPs was determined using the following formulae:

$$
\begin{aligned}
& \text { Encapsulation } \\
& \text { efficiency }(\mathrm{EE} \%)
\end{aligned}=\frac{\begin{array}{c}
\text { Amount of DEX } \\
\text { encapsulated in NP }
\end{array}}{\begin{array}{c}
\text { Amount of DEX added in } \\
\text { the dispersion }
\end{array}} \times 100 \%
$$$$
\underset{(\mathrm{DL} \%)}{\text { Drug loading }}=\frac{\text { Amount of DEX in NP }}{\text { NP weight containing DEX }} \times 100 \%
$$

\section{In vitro release of DEX from DEX-NPs}

The in vitro release of DEX from NPs was examined under three different media conditions ( $\mathrm{pH}$ 7.4 PBS, artificial perilymph [AP], rat plasma) at $37^{\circ} \mathrm{C}$ to evaluate the influence of media on the rate of release. Briefly, $20 \mathrm{mg}$ of DEX-NPs were added to a centrifuge tube and suspended in $5 \mathrm{~mL}$ of release medium containing $0.1 \%$ Tween- 80 (to maintain the sink condition, by increasing the solubility of DEX in the medium and avoiding the binding of DEX to the tube wall). Next, the solution was stirred at $110 \mathrm{rpm}$ in the gas bath at $37^{\circ} \mathrm{C}$. At designated time points, the tubes were centrifuged $(11,000 \times g, 30$ minutes) and the supernatants containing DEX were transferred to empty tubes for further analyses. The DEX-NPs in the pellet were resuspended in $5 \mathrm{~mL}$ of fresh medium to continue the release studies.

Then, concentrations of DEX were determined using high-pressure liquid chromatography (HPLC) combined with mass spectrometry (LC/MS/MS). Samples were prepared by extracting $5 \mu \mathrm{L}$ of the release medium using $100 \mu \mathrm{L}$ of dichloromethane:hexane:methyl tert-butyl ether $(1: 1: 1, \mathrm{v} / \mathrm{v} / \mathrm{v})$. The extraction step was repeated once. The organic fraction was isolated, dried, and reconstituted with $100 \mu \mathrm{L}$ of a water/methanol solution $(1 / 1, \mathrm{v} / \mathrm{v})$. For HPLC, a $25 \mu \mathrm{L}$ sample was loaded on a Hypersil GOLD ${ }^{\mathrm{TM}} \mathrm{C} 18$ reverse-phase column $(2.1 \mathrm{~mm} \times 100 \mathrm{~mm} ; 3 \mu \mathrm{m}$ particle size; Thermo Fisher Scientific) equipped with a Thermo Fisher Scientific TSQ Quantum ${ }^{\text {TM }}$ Access MAX LC/MS/MS system (Thermo Fisher Scientific). The gradient elution was set with a combination of water with $0.13 \%$ formic acid and acetonitrile in a 17-minute program. The mobile phase was initiated with a flow rate of $0.2 \mathrm{~mL} /$ minute. MS analysis was performed on a Thermo Fisher Scientific TSQ Quantum ${ }^{\mathrm{TM}}$ mass spectrometer with an electrospray ionization probe. The detection limit for DEX in this assay was $1 \mathrm{ng} / \mathrm{mL}$. DEX concentrations were interpolated using equations derived from calibration curves.

\section{In vivo biodistribution and in vivo release} of DEX-NPs following the RWM administration Administration of DEX-NPs onto RWM

Guinea pigs were anesthetized with an intraperitoneal (IP) injection of $1 \%$ pentobarbital sodium (35 mg/kg). During the procedure, the animals were placed on a heating pad $\left(38^{\circ} \mathrm{C}\right)$. A subcutaneous injection of $1 \%$ lidocaine was performed to reduce pain. A postauricular incision was made and the muscle was dissociated via blunt dissection and retracted until exposure of the auditory bulla. A hole of 2-3 $\mathrm{mm}$ in diameter was drilled on the bulla to provide 
direct visualization of the round window niche. A DEX or DEX-NPs solution $(5 \mu \mathrm{L}, 10 \mathrm{mg} / \mathrm{mL})$ was applied onto the RWM using a microsyringe. The guinea pigs were fixed at this position for 30 minutes. The bulla opening was sealed with dental cement and the incision was closed with sutures.

\section{In vivo biodistribution of Coumarin 6-labeled NPs following the RWM administration}

To determine the distribution of NPs in the inner ear after RWM delivery, we labeled the NPs with a fluorescent labeling dye, Coumarin 6. Guinea pigs ( $n=3$ in each group) were randomly assigned into two groups for the RWM administration of Coumarin $6(5 \mu \mathrm{L}, 25 \mu \mathrm{g} / \mathrm{mL})$ and Coumarin 6-labeled NPs $(5 \mu \mathrm{L}, 25 \mu \mathrm{g} / \mathrm{mL})$. All guinea pigs were sacrificed 1 hour after RWM drug administration via cervical dislocation. Cochleae were harvested from the animals, immersed in 4\% paraformaldehyde in PBS ( $\mathrm{pH} 7.4$ ) for 2 hours, and decalcified in $0.1 \mathrm{M}$ ethylenediaminetetraacetic acid (EDTA) in PBS ( $\mathrm{pH} \mathrm{7.4)} \mathrm{for} 14$ days at room temperature. The tissues were embedded in optimal cutting temperature and cut into $10 \mu \mathrm{m}$ thick cryosections. Midmodiolus sections from the cochlea of each animal were used for histological analysis. Nuclei were stained with DAPI. The specimens were examined under a fluorescence microscope (Leica Microsystems, Wetzlar, Germany).

\section{In vivo release of DEX-NPs following the RWM administration}

To assess the sustained release of DEX-NPs in vivo, the DEX concentration in cochlea following the administration of a single dose of DEX-NPs onto RWM was examined. The DEX concentration of either directly used (DEX solution) or in the DEX-NP solution was $10 \mathrm{mg} / \mathrm{mL}$ and $5 \mu \mathrm{L}$ of the solution was administered onto RWM in each animal ( $n=5$ for each time point). At the designed time points ( 1 hour, 3 hours, 6 hours, 12 hours, 24 hours, and 48 hours after drug administration), the cochleae were harvested under general anesthesia and perilymph was collected.

To avoid contamination by the cerebrospinal fluid influx after perforation of the cochlear shell, driven by hydrostatic pressure, perilymph was collected ex vivo. Briefly, animals were decapitated under deep anesthesia with an overdose of pentobarbital. The temporal bone was rapidly removed and the cochleae were then dissected from the temporal bones. A small hole was made at the apex, and 5-7 $\mu \mathrm{L}$ of perilymph was sampled using a microsyringe pump (Micro4; World Precision Instruments, Sarasota, FL, USA). All samples were stored at $-80^{\circ} \mathrm{C}$ for further analysis. DEX in perilymph was quantified using LC/MS/MS, as described earlier. The detection limit for DEX in this assay was $1 \mathrm{ng} / \mathrm{mL}$.

\section{Functional and histological assessments}

Establishment of a hearing loss animal model

Guinea pigs were treated with cisplatin to induce hearing loss. Specially, 18 guinea pigs were assigned to three groups ( $n=6$ per group), and all the animals received a single-dose IP injection of $10 \mathrm{mg} / \mathrm{kg}, 12 \mathrm{mg} / \mathrm{kg}$, and $15 \mathrm{mg} / \mathrm{kg}$ cisplatin. Auditory brainstem response (ABR) thresholds were measured at frequencies of $4 \mathrm{kHz}, 8 \mathrm{kHz}, 16 \mathrm{kHz}$, and $24 \mathrm{kHz}$ 1 day before and 3 days after treatment with cisplatin. The optimal ototoxic dose of cisplatin was considered to have desired ototoxicity and low mortality rates.

\section{Evaluation of otoprotective effects of DEX-NPs drug administration}

The otoprotective effects of DEX and DEX-NPs against cisplatin toxin were evaluated in guinea pigs. A total of 52 guinea pigs were divided into one of seven groups: 1) saline $(n=6) ; 2) \operatorname{CDDP}(n=8) ; 3) \operatorname{CDDP}+\operatorname{NPs}(n=8)$; 4) $\operatorname{DEX}(n=6)$; 5) DEX-NPs $(n=6)$; 6) CDDP + DEX $(n=8)$; and 7) CDDP + DEX-NPs ( $n=10)$. CDDP was administered IP at a dose of $12 \mathrm{mg} / \mathrm{kg}$ (the dose had been optimized). Then, $5 \mu \mathrm{L}$ of saline, NPs $(5 \mu \mathrm{g} / \mathrm{mL}), \operatorname{DEX}(10 \mathrm{mg} / \mathrm{mL})$ or DEX-NPs (10 mg/mL) were applied onto the RWM 1 hour before CDDP injection. ${ }^{23}$ ABR thresholds were measured at frequencies of $4 \mathrm{kHz}, 8 \mathrm{kHz}, 16 \mathrm{kHz}$, and $24 \mathrm{kHz} 1$ day before and 3 days after drug administration.

\section{Evaluation of otoprotective effects of DEX-NPs (ABR)}

ABR tests were conducted at specific frequencies using the ABR workstation from Tucker-Davis Technologies Inc (Alachua, FL, USA). ABR was recorded with a subcutaneously implanted sterile stainless steel electrode. The active electrode was placed at the forehead, the reference electrode at the mastoid of the measured ear, and the ground electrode at the contralateral hind leg. Animals with normal external auditory canal and tympanic membrane were examined using an otoscope before the ABR measurements were conducted. Animals were anesthetized with an IP injection of $1 \%$ pentobarbital sodium ( $35 \mathrm{mg} / \mathrm{kg}$ ). During the procedure, the animal was placed on a heating pad $\left(38^{\circ} \mathrm{C}\right)$ in a soundproof chamber. Pure tone bursts at $4 \mathrm{kHz}, 8 \mathrm{kHz}, 16 \mathrm{kHz}$, and $24 \mathrm{kHz}$ were generated digitally for a duration of $10 \mathrm{~ms}$ and with a rise-fall time of $1 \mathrm{~ms}$. Acoustic stimuli were presented at a rate of 21.1 per second into the external auditory canal of the animal. The evoked potentials were filtered with a bandpass 
filter between $100 \mathrm{~Hz}$ and 3,000 Hz, and averaged 512 times.

For each given frequency, the tone burst intensity started at a $90 \mathrm{~dB}$ sound pressure level for the pretreatment group and at a $110 \mathrm{~dB}$ sound pressure level for the post-treatment group, and it decreased in steps of $5 \mathrm{~dB}$ until the threshold was reached. The ABR threshold was defined as the lowest intensity that a reproducible wave III could be recorded.

\section{Evaluation of otoprotective effects of DEX-NPs hair cell counting}

Three days after drug administration, guinea pigs were anesthetized with lethal doses of $1 \%$ pentobarbital sodium and the cochleae were harvested and perfused with $4 \%$ paraformaldehyde in PBS and further fixed with 4\% paraformaldehyde for 4 hours at room temperature. After decalcification with $0.1 \mathrm{M}$ EDTA for 14 days, the organs of Corti were microdissected under a microscope as a surface preparation. The hair cells were evaluated on the animals in four groups: saline; CDDP; CDDP + DEX; and CDDP + DEX-NPs ( $n=6$ per group). DEX $(5 \mu \mathrm{L}, 10 \mathrm{mg} / \mathrm{mL})$ or DEX-NPs were applied onto the RWM 1 hour before CDDP injection. ${ }^{23}$ F-actin was stained with rhodamine phalloidin (1:100) to identify cochlear hair cells. Hair cells were examined under a Leica TCS SPE confocal microscope (Leica Microsystems). The residual outer hair cells (OHCs) were counted in a $1 \mathrm{~mm}$ long strip at the region, approximately $60 \%$ from the apex.

\section{Inflammatory responses to DEX-NPs}

Inflammatory reactions upon DEX-NPs administration on RWM ( $5 \mu \mathrm{L}, 10 \mathrm{mg} / \mathrm{mL}$ ) were assessed using hematoxylin and eosin staining. Briefly, six guinea pigs were randomly assigned into two groups ( $\mathrm{n}=3$ per group): 1) saline $(5 \mu \mathrm{L})$ RWM; and 2) DEX-NPs ( $5 \mu \mathrm{L}, 10 \mathrm{mg} / \mathrm{mL})$ RWM. Three days after drug administration, guinea pigs were anesthetized with lethal doses of $1 \%$ pentobarbital sodium. Tympanic bullae were removed, fixed in $4 \%$ buffered paraformaldehyde for 2 hours, and decalcified in 0.1 M EDTA for 14 days. The cochleae were trimmed and embedded for modiolus section. Then, $5 \mu \mathrm{m}$ sections were cut, stained with hematoxylin and eosin, and examined via light microscopy.

\section{Statistical analysis}

All statistical analyses were performed using GraphPad Prism 5.0 software (GraphPad Software, Inc., La Jolla, CA, USA). Differences between groups were examined using Student's $t$-test or analysis of variance with Turkey's multiple comparison tests. Statistical significance was set as $P$-values $<0.05$.

\section{Results Physical characterization of DEX-NPs}

DEX-NPs were prepared using an emulsion and evaporation technique, as reported previously. ${ }^{22}$ DEX-NPs were spherical in shape with a hydrophilic polyethylene glycol (PEG) shell and a hydrophobic polylactic acid core. DEX was loaded into spherical structures covered with PEG to improve the water solubility of the drug (Figure 2A). The average particle size, zeta potential, DL, and EE of DEX-NPs were shown in Table 1. The representative TEM and AFM images of DEX-NPs revealed that NPs were spherical in shape with a smooth surface and no aggregation or adhesion. The particle sizes of DEX-NPs determined by TEM, AFM, and DLS were $123.47 \pm 7.88 \mathrm{~nm}, 110.37 \pm 3.36 \mathrm{~nm}$, and $130 \pm 4.78 \mathrm{~nm}$ (polydispersity index $=0.098$ ), respectively (Figure $2 \mathrm{~B}, \mathrm{C}$ and D). The three sizes of DEX-NPs were determined in the same range, which is consistent with previous findings. ${ }^{22}$ The surface of DEX-NPs was negatively charged, as determined by the zeta potential $(-26.13 \pm 3.28 \mathrm{mV})$. DL and EE were $8.24 \% \pm 2.85 \%$ and $48.58 \% \pm 6.74 \%$, respectively.

\section{In vitro release of DEX-NPs}

The in vitro release of DEX from DEX-NPs was examined in PBS (pH 7.4), AP ( $\mathrm{pH} 7.4$ ), and rat plasma (Figure 2E). In PBS and AP, DEX was released in a biphasic pattern; specifically, there was an initial rapid release followed by a slower and sustained release. In PBS medium, the accumulated release of DEX from DEX-NPs was 34.08\% $03.08 \%$ on day 1 and $78.01 \% \pm 6.00 \%$ on day 23 . In contrast, in AP medium, the accumulated release of DEX from DEX-NPs was $68.14 \% \pm 4.35 \%$ on day 1 and $93.42 \% \pm 5.43 \%$ on day 13 . DEX release from NPs in rat plasma was significantly faster compared to that in PBS and AP $(P<0.05,84.43 \% \pm 4.29 \%$ on day 1). This may be the result of increased drug diffusion speed induced by plasma enzyme-catalyzed hydrolysis of the polymer matrix. Similar patterns have been previously reported for the release of paclitaxel from other PEG-PLA NP systems. ${ }^{22}$

\section{In vivo distribution of Coumarin 6-labeled NPs in cochleae}

Coumarin 6, a fluorescence dye, is commonly used to trace the distribution of NPs. Coumarin 6-labeled NPs are stable in PBS medium with only 5\% Coumarin 6 released from the NPs at a long period incubation in PBS ( $\mathrm{pH} 7.4) .{ }^{24} \mathrm{We}$ followed Coumarin 6 in cochlea.

After the administration of free Coumarin 6 to the RWM in guinea pigs, weak fluorescence was observed in the cochlea 


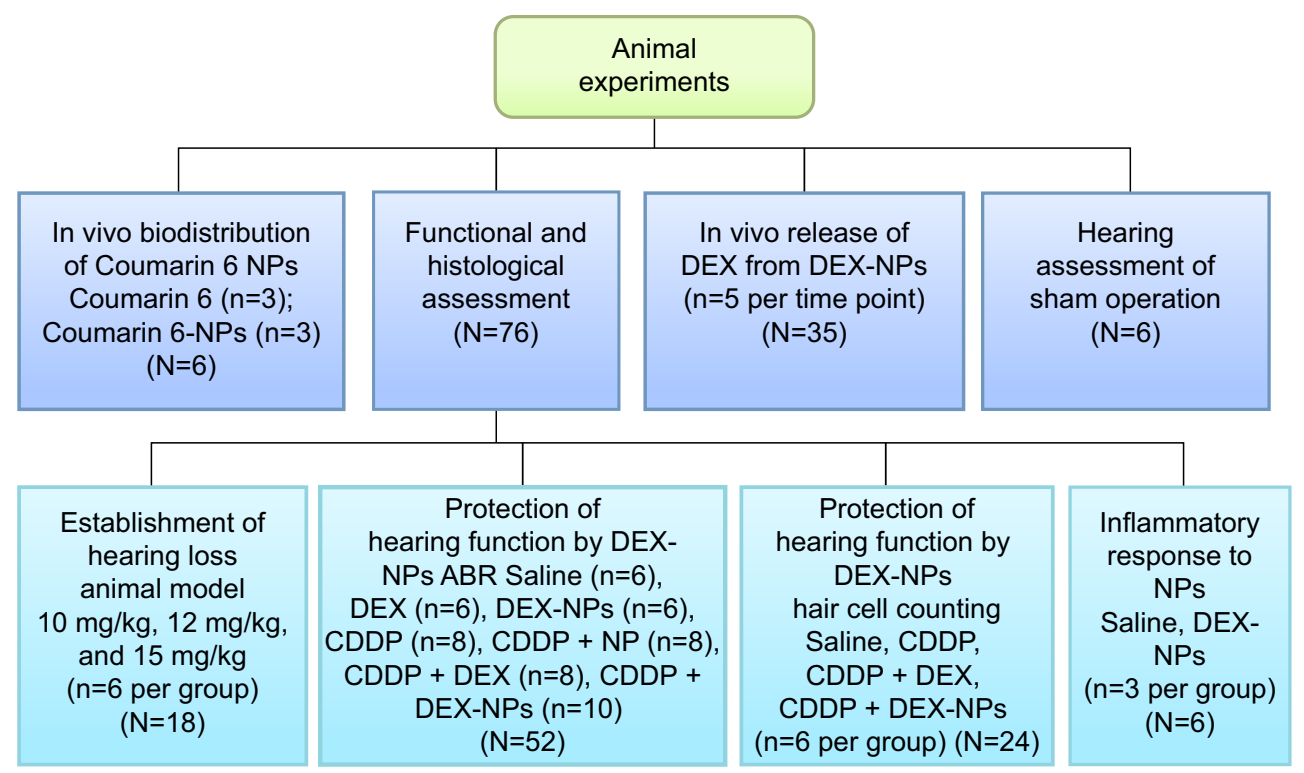

Figure I Outline of animal experiments.

Notes: There were seven animal experiments - namely, the in vivo distribution of Coumarin $6 \mathrm{NPs}(\mathrm{n}=6)$, the in vivo release of DEX from DEX-NPs ( $\mathrm{N}=35)$, the hearing assessment of the sham operation $(\mathrm{N}=6)$, the establishment of a hearing loss animal model $(\mathrm{N}=18)$, the protection of hearing function by $\mathrm{DEX}-\mathrm{NPs}-\mathrm{ABR}(\mathrm{N}=52)$, the protection of hearing function by DEX-NPs - hair cell counting ( $\mathrm{N}=24)$, and inflammatory response to NPs $(\mathrm{N}=6)$.

Abbreviations: NP, nanoparticle; n, number; DEX, dexamethasone; DEX-NPs, dexamethasone-loaded polyethylene glycol-coated polylactic acid stealth nanoparticles; ABR, auditory brainstem response; CDDP, cis-diamminedichloroplatinumll.
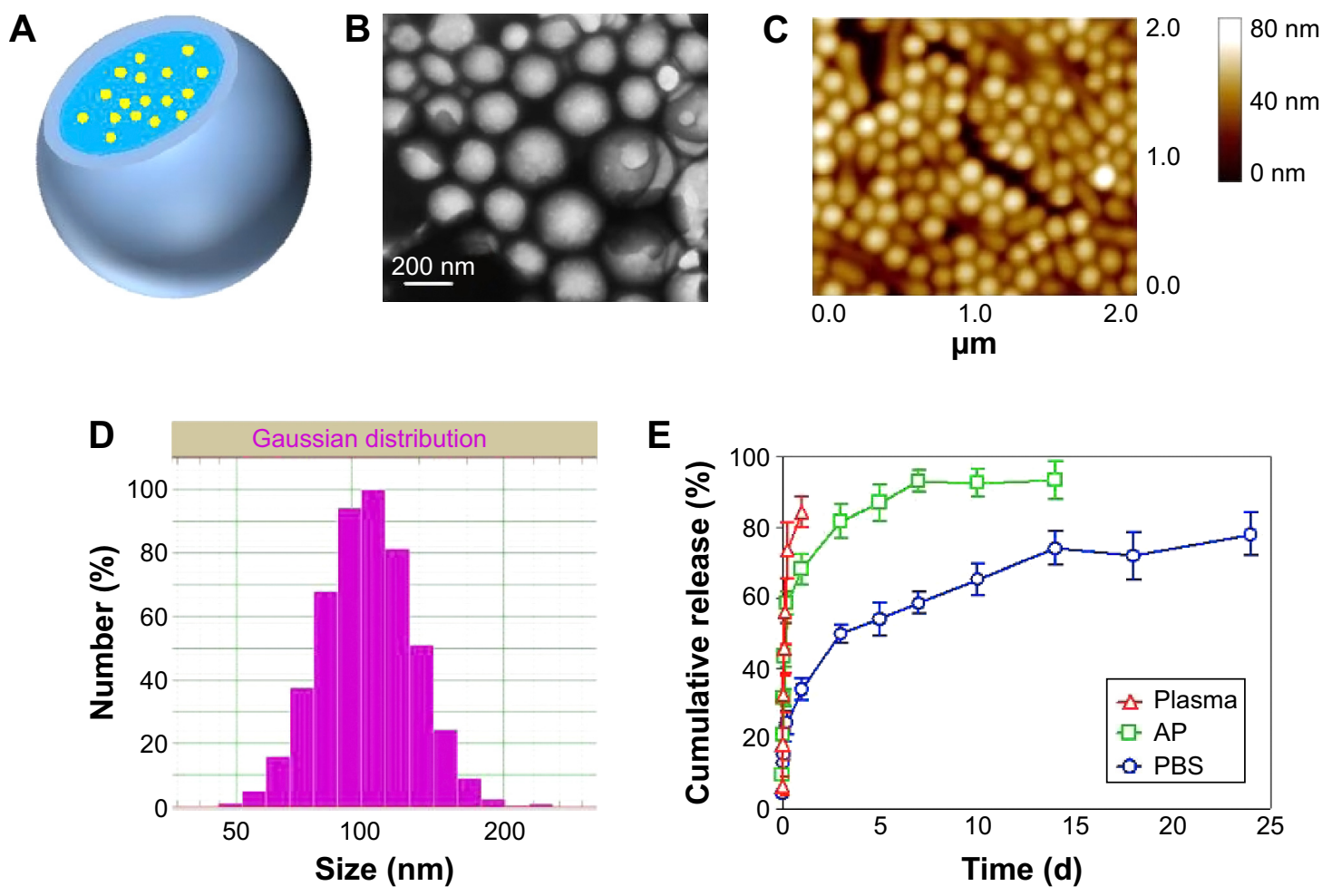

Figure 2 Characterization of DEX-NPs.

Notes: (A) Fabrication of DEX-NPs. Schematic representation of the nanoparticle structure: DEX (yellow balls) was encapsulated in the nanoparticles (light blue sphere) modified with PEG (gray ball shell). (B) Representative transmission electron microscopy image. (C) Two-dimensional nanoparticle image of atomic force microscopy. (D) Size distribution determined by dynamic light scattering. (E) In vitro release of DEX from DEX-NPs in PBS (pH 7.4), rat plasma, and AP (pH 7.4) (mean \pm SD; $n=3$ ).

Abbreviations: AP, artificial perilymph; PBS, phosphate buffered saline; PEG, polyethylene glycol; d, days; DEX-NPs, dexamethasone-loaded polyethylene glycol-coated polylactic acid stealth nanoparticles; DEX, dexamethasone; SD, standard deviation; n, number. 
Table I Physicochemical characteristics of DEX-NPs

\begin{tabular}{|c|c|c|c|c|c|c|}
\hline \multirow[t]{2}{*}{ Nanoparticles } & \multicolumn{3}{|c|}{ Particle size $(\mathrm{nm})$} & \multirow{2}{*}{$\begin{array}{l}\text { Zeta potential } \\
(\mathrm{mV})\end{array}$} & \multirow[t]{2}{*}{$D^{d}(\%)$} & \multirow[t]{2}{*}{$\mathrm{EE}^{\mathrm{e}}(\%)$} \\
\hline & $\operatorname{DLS}^{\mathrm{a}}(\mathrm{PI})$ & TEM ${ }^{\mathrm{b}}$ & AFMc $^{c}$ & & & \\
\hline DEX-NPs & $130 \pm 4.78$ & $123.47 \pm 7.88$ & $110.37 \pm 3.36$ & $-26.13 \pm 3.28$ & $8.24 \pm 2.85$ & $48.58 \pm 6.74$ \\
\hline
\end{tabular}

Notes: ${ }^{a} D L S(n=6)$. ${ }^{\circ} T E M(n=6) .{ }^{c} A F M(n=6)$. ${ }^{d} D L(n=3)$. ${ }^{e} E E(n=3)$. All results are expressed as the mean $\pm S D$.

Abbreviations: DEX-NPs, dexamethasone-loaded polyethylene glycol-coated polylactic acid stealth nanoparticles; DLS, dynamic light scattering; PI, polydispersity index; TEM, transmission electron microscopy; AFM, atomic force microscopy; DL, drug loading; EE, encapsulation efficiency; n, number; SD, standard deviation.

after 1 hour (Figure 3A). In contrast, in guinea pigs treated with Coumarin 6-labeled NPs, a strong green fluorescence region was visible in the cochlea 1 hour after administration (Figure 3B). Green fluorescence was observed in the stria vascularis and organs of Corti in all the cochlear turns, as well as in the cochlear modiolus. These results suggest that PEG-PLA NPs encapsulate drugs effectively and adhere on and penetrate the RWM.

\section{In vivo release of DEX-NPs following the RWM administration}

Concentration-time courses for DEX and DEX-NPs after RWM delivery to guinea pigs are shown in Figure 4. Specifically, we assessed DEX concentrations in cochlear perilymph at 1 hour, 3 hours, 6 hours, 12 hours, 24 hours, and 48 hours after RWM administration of DEX or DEX-NPs.

At 1 hour after RWM administration, the DEX that diffused into the inner ear following free DEX treatment $(12,717.91 \pm 6,550.95 \mathrm{ng} / \mathrm{mL})$ was significantly higher than that of the DEX-NP treatment $(8,316.217 \pm 2,782.65 \mathrm{ng} / \mathrm{mL})$. The concentration of free DEX was significantly reduced to $864.24 \pm 407.11 \mathrm{ng} / \mathrm{mL}$ after 6 hours and was below the detection limit at 12 hours. In contrast, DEX concentrations in the cochleae of DEX-NPs treated with animals still remained high after 24 hours $(1,376.21 \pm 1,234.94 \mathrm{ng} / \mathrm{mL})$ and 48 hours (269.85 $\pm 161.01 \mathrm{ng} / \mathrm{mL})$.

\section{Establishment of a hearing loss animal model}

The ABR technique was used to monitor auditory function. All guinea pigs treated with cisplatin $(10 \mathrm{mg} / \mathrm{kg}$ and $12 \mathrm{mg} / \mathrm{kg}$ ) experienced a statistically significant threshold elevation by day 3 at all detected frequencies except for $4 \mathrm{kHz}$ with $10 \mathrm{mg} / \mathrm{kg}$ cisplatin (Figure $5 \mathrm{~A} ; P<0.05$ ). For $10 \mathrm{mg} / \mathrm{kg}$ of cisplatin, the mean ABR threshold shifts exhibited no significant differences among the detected frequencies (Figure 5B). A cisplatin dose response was observed with average threshold shifts of $23.59 \pm 4.68 \mathrm{~dB}$ for $10 \mathrm{mg} / \mathrm{kg}$ and $69.69 \pm 5.01 \mathrm{~dB}$ for $12 \mathrm{mg} / \mathrm{kg}$ across all frequencies. In addition, the average threshold shifts induced by $12 \mathrm{mg} / \mathrm{kg}$ cisplatin were significantly higher than those induced by $10 \mathrm{mg} / \mathrm{kg}$ across all frequencies $(P<0.001)$.

Lethality rates in guinea pigs at day 3 after treatment with $10 \mathrm{mg} / \mathrm{kg}$ and $12 \mathrm{mg} / \mathrm{kg}$ of cisplatin were $0 \%$ and $25 \%$, respectively. In addition, the lethality rate in guinea pigs at day 3 after treatment with $15 \mathrm{mg} / \mathrm{kg}$ cisplatin was $80 \%$. Therefore, cisplatin of $12 \mathrm{mg} / \mathrm{kg}$ was selected for the following experiments.
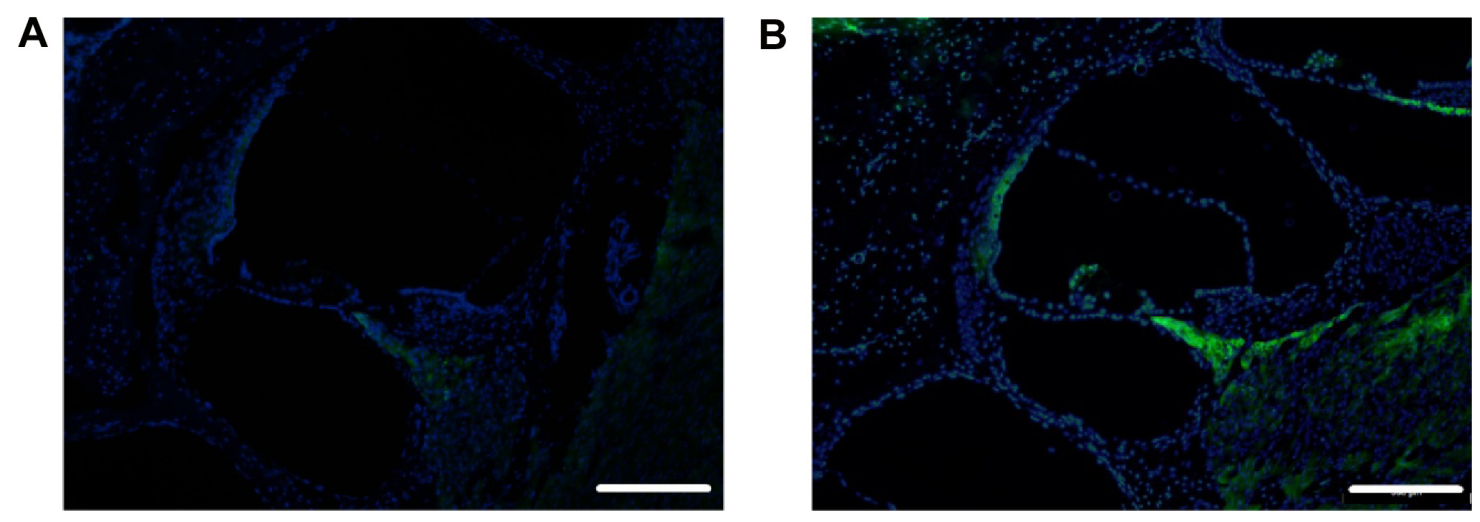

Figure 3 Coumarin 6-labeled NPs distribution in the cochlea via RWM administration at I hour after drug exposure.

Notes: (A) Weak fluorescence was detected in the cochlear modiolus after free Coumarin 6 RWM administration. Scale bar, $500 \mu \mathrm{m}$. Nuclei were stained with DAPI (blue). (B) Strong green fluorescence regions were visible in the stria vascularis and organs of Corti in all the cochlear turns, as well as in the spiral ganglion cells after Coumarin 6 NPs RWM administration.

Abbreviations: NP, nanoparticle; RWM, round window membrane; DAPI, 4'6-diamidino-2-phenylindole. 


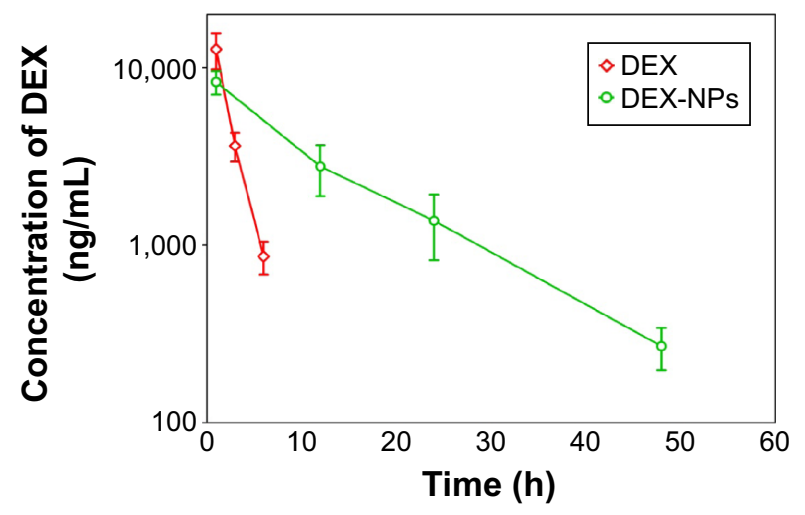

Figure 4 In vivo DEX concentrations in cochlear perilymph via RWM administration of free DEX or DEX-NPs.

Note: Data are presented as the mean $\pm \operatorname{SEM}(n=5)$.

Abbreviations: DEX, dexamethasone; DEX-NPs, dexamethasone-loaded polyethylene glycol-coated polylactic acid stealth nanoparticles; h, hours; RWM, round window membrane; SEM, standard error of the mean; $n$, number.

\section{Evaluation of otoprotective effects of DEX-NPs - assessment of ABR}

Following the sham operation, we observed no significant difference between day 0 , day 1 , and day 3 after RWM administration at all tested frequencies (Figure 6). This result showed that the procedure of RWM administration of saline had no influence on the guinea pigs' hearing function.

The results of the ABR tests indicated that the cisplatininduced hearing loss was partially protected by DEX-NPs (Figure 7). In the control guinea pigs treated with saline, DEX and DEX-NPs did not change ABR thresholds at any tested frequency, indicating that the concentration of DEX was nontoxic and that the PEG-PLA polymer was biocompatible (Figures 7A and B). This result was consistent with data from Buckiová et al's study. ${ }^{21}$

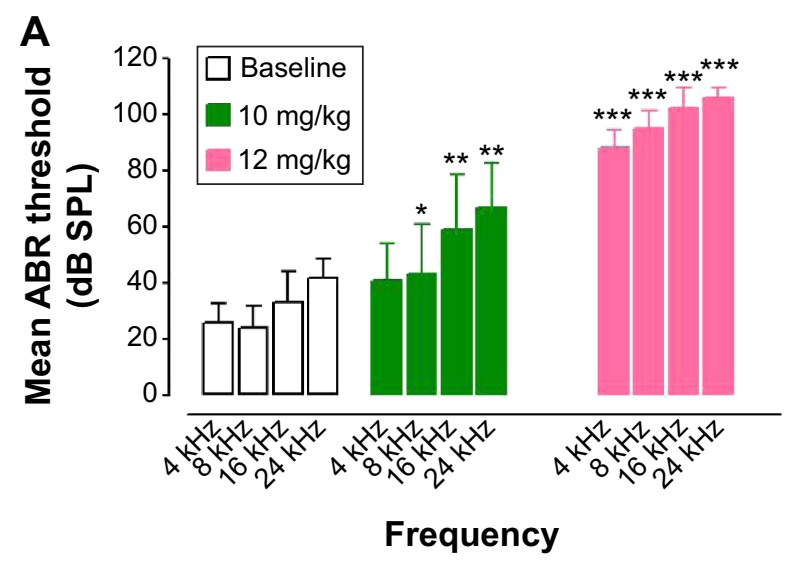

The baseline of CDDP, CDDP + NPs, CDDP + DEX, and CDDP + DEX-NPs were measured at 1 day before treatment and showed no significant difference (Figure 7C). However, at day 3 after drug administration, the mean ABR threshold of CDDP + DEX-NPs $(63.5 \pm 15.75 \mathrm{~dB}$ at $4 \mathrm{kHz}$ and $69.00 \pm 17.68 \mathrm{~dB}$ at $8 \mathrm{kHz}$ ) were significantly lower as compared with those obtained with CDDP $(85.63 \pm 11.78 \mathrm{~dB}$ at $4 \mathrm{kHz}$ and $88.13 \pm 9.98 \mathrm{~dB}$ at $8 \mathrm{kHz} ; P<0.01$ and $P<0.05$, respectively) and CDDP $+\operatorname{DEX}(81.88 \pm 6.51 \mathrm{~dB}$ at $4 \mathrm{kHz}$ and $89.38 \pm 6.23 \mathrm{~dB}$ at $8 \mathrm{kHz} ; P<0.05$ and $P<0.01$, respectively). The mean $\mathrm{ABR}$ threshold of CDDP + DEX exhibited no significant difference when compared with that of the CDDP group.

In the deafened guinea pigs treated with CDDP + DEX-NPs, ABR threshold shifts were $42.00 \pm 19.61 \mathrm{~dB}$ at $4 \mathrm{kHz}$ and $48.50 \pm 22.74 \mathrm{~dB}$ at $8 \mathrm{kHz}$ (Figure 7D), which were significantly lower when compared to those obtained with CDDP (57.50 $\pm 10.35 \mathrm{~dB}$ at $4 \mathrm{kHz}$ and $63.13 \pm 8.42 \mathrm{~dB}$ at $8 \mathrm{kHz} ; P<0.05$ and $P<0.05$, respectively) and CDDP + DEX $(57.5 \pm 5.98 \mathrm{~dB}$ at $4 \mathrm{kHz}$ and $67.5 \pm 8.02 \mathrm{~dB}$ at $8 \mathrm{kHz}$; $P<0.05$ and $P<0.01$, respectively) However, the CDDP + DEX group showed no significant differences in the ABR threshold shift, in contrast to the CDDP treatment group at all detected frequencies.

Taken together, DEX-NPs showed a significant protection effect against cisplatin-induced hearing loss at $4 \mathrm{kHz}$ and $8 \mathrm{kHz}$, but not with free DEX.

\section{Evaluation of otoprotective effects of DEX-NPs - assessment of hair cell loss}

In guinea pigs, $6 \mathrm{kHz}$ locates at approximately $50 \%$ from the apex of the cochlea along the longitude of the cochlea. ${ }^{25}$ According to the $\mathrm{ABR}$ results, the protected frequencies were

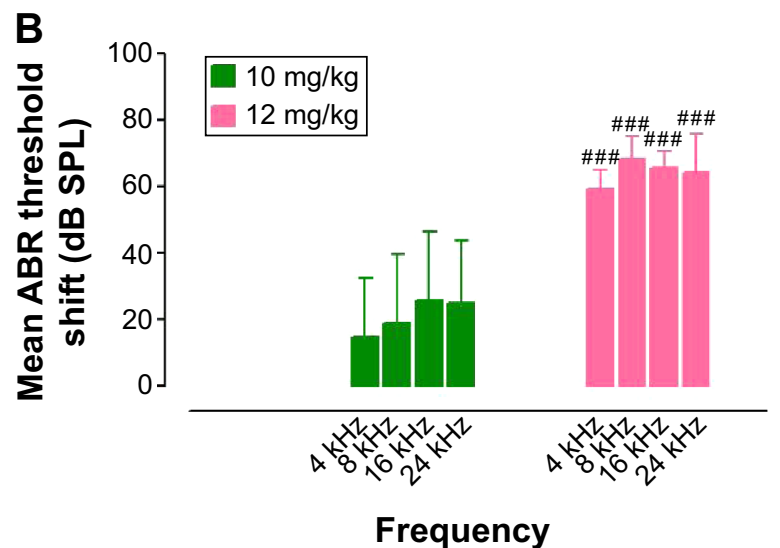

Figure 5 Establishment of cisplatin-induced hearing loss animal models.

Notes: Mean (A) ABR threshold and (B) shift per frequency for guinea pigs 3 days after treatment with $10 \mathrm{mg} / \mathrm{kg}$ and $12 \mathrm{mg} / \mathrm{kg}$ of intraperitoneal cisplatin ( $\mathrm{n}=6$ ). $* \mathrm{P}<0.05$, $* * P<0.0 \mathrm{l}$, and $* * * P<0.00 \mathrm{I}$ as compared with baseline. $P<0.00 \mathrm{l}$ as compared with the $10 \mathrm{mg} / \mathrm{kg}$ group.

Abbreviations: ABR, auditory brainstem response; SPL, sound pressure level; $n$, number. 


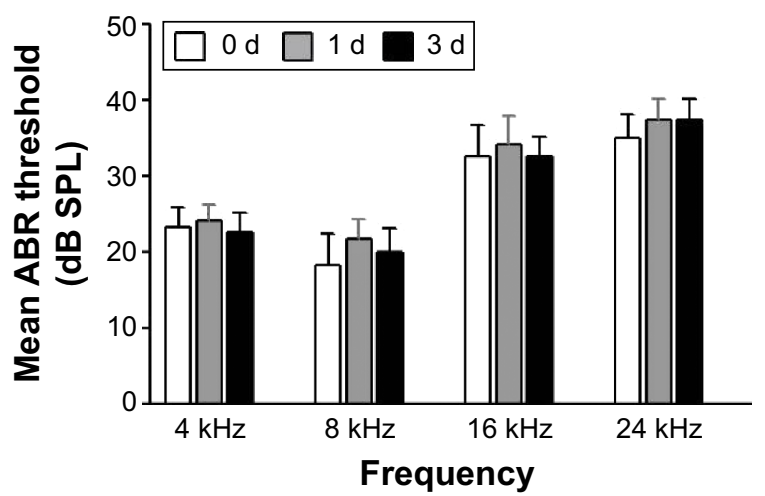

Figure 6 Average $A B R$ threshold (mean $\pm S D ; n=6$ ) for each frequency prior to $(0 \mathrm{~d})$ and after ( $\mathrm{d}$ and $3 \mathrm{~d}$ ) the sham operation of the left ear.

Abbreviations: ABR, auditory brainstem response; SPL, sound pressure level; $d$, days; SD, standard deviation; $n$, number.

$4 \mathrm{kHz}$ and $8 \mathrm{kHz}$. To correlate the auditory function to the number of hair cells, we counted the hair cells in the region about $60 \%$ from the apex.

As shown in Figure 8A, there was cisplatin-induced severe loss of OHCs and abnormalities as compared to the control animals. The administration of DEX-NPs 1 hour before cisplatin treatment largely preserved hair cells from cisplatininduced damage, whereas the administration of free DEX did not show such a protective effect. As shown in Figure 8B, quantification of the $\mathrm{OHC}$ number at the approximately $60 \%$ region from the apex region showed significantly more $\mathrm{OHCs}$ in the DEX-NPs-treated animals than in the free DEX group or no-treatment group $(P<0.001$ and $P<0.001$, respectively). The survival number of OHCs treated with CDDP + DEX-NPs was significantly higher than those treated with CDDP and CDDP + DEX ( $P<0.001$ and $P<0.001$, respectively). The number of normal OHCs treated with CDDP + DEX-NPs was significant higher than that of the groups treated with CDDP and CDDP + DEX $(P<0.001$ and $P<0.05$, respectively). However, the CDDP + DEX group exhibited no protective effects against cisplatin on hair cell survival when compared with the CDDP group. Our results indicate that DEX-NPs have greater therapeutic potential when protecting hair cells against cisplatininduced damage than free DEX.
A

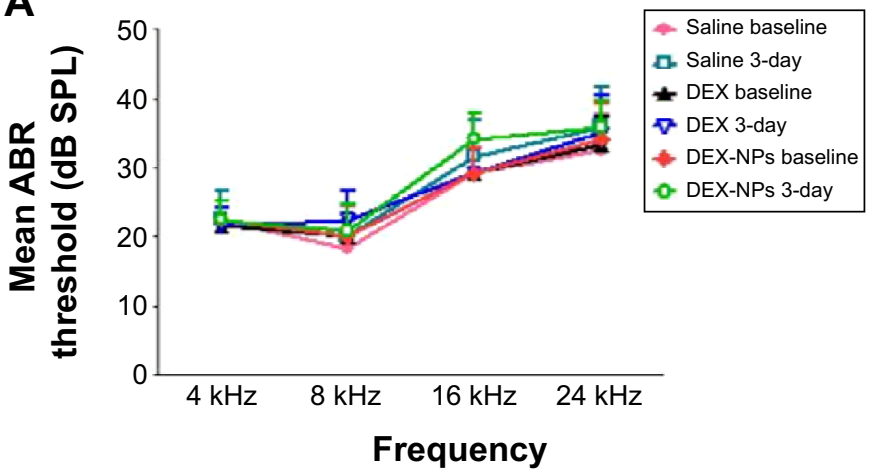

C

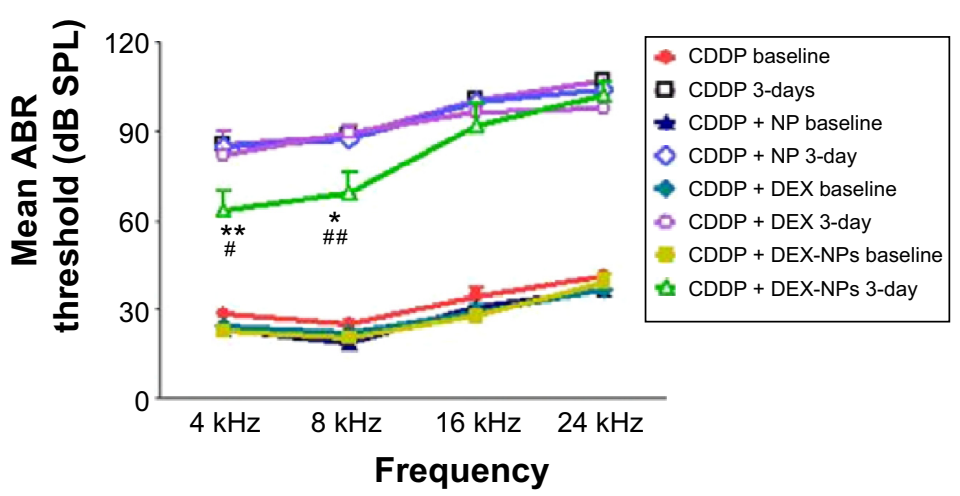

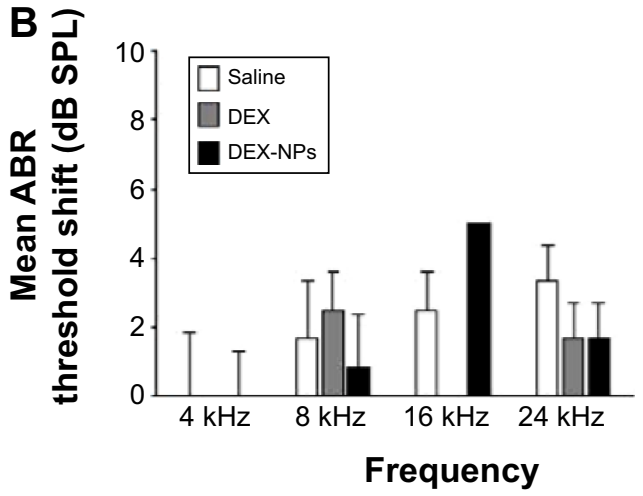

D

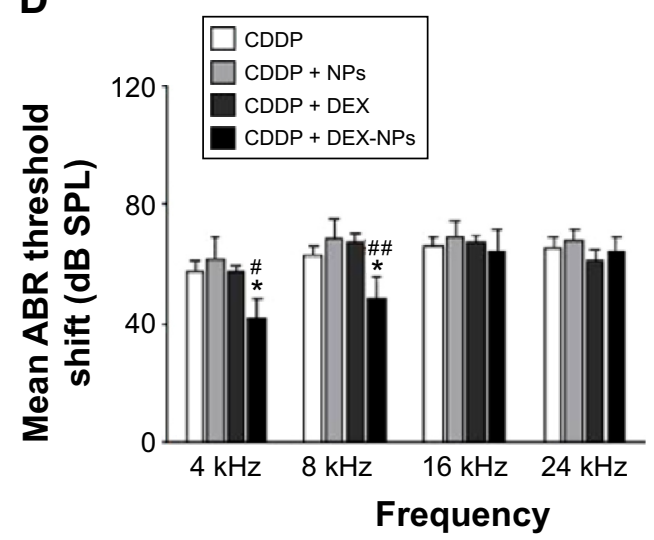

Figure 7 Mean ABR threshold and shift of guinea pigs treated with saline, DEX, DEX-NPs, CDDP, CDDP + NPs, CDDP + DEX, and CDDP + DEX-NPs by RWM administration.

Notes: Mean (A and C) ABR threshold and (B and $\mathbf{D})$ shift of guinea pigs treated with saline $(n=6), \mathrm{DEX}(\mathrm{n}=6), \mathrm{DEX}-\mathrm{NPs}(\mathrm{n}=6), \mathrm{CDDP}(\mathrm{n}=8), \mathrm{CDDP}+\mathrm{NPs}(\mathrm{n}=8), \mathrm{CDDP}+$ $\operatorname{DEX}(n=8)$, and CDDP + DEX-NPs $(n=10)$ by RWM administration. ABR shift thresholds are given as the mean $\pm S D$. $* P<0.05$ and $* * P<0.01$ as compared with CDDP; ${ }^{\#}<<0.05$ and ${ }^{\# P}<0.01$ as compared with CDDP + DEX.

Abbreviations: ABR, auditory brainstem response; SPL, sound pressure level; DEX, dexamethasone; DEX-NPs, dexamethasone-loaded polyethylene glycol-coated polylactic acid stealth nanoparticles; CDDP, cis-diamminedichloroplatinumll; NP, nanoparticle; RWM, round window membrane; SD, standard deviation; n, number. 
A
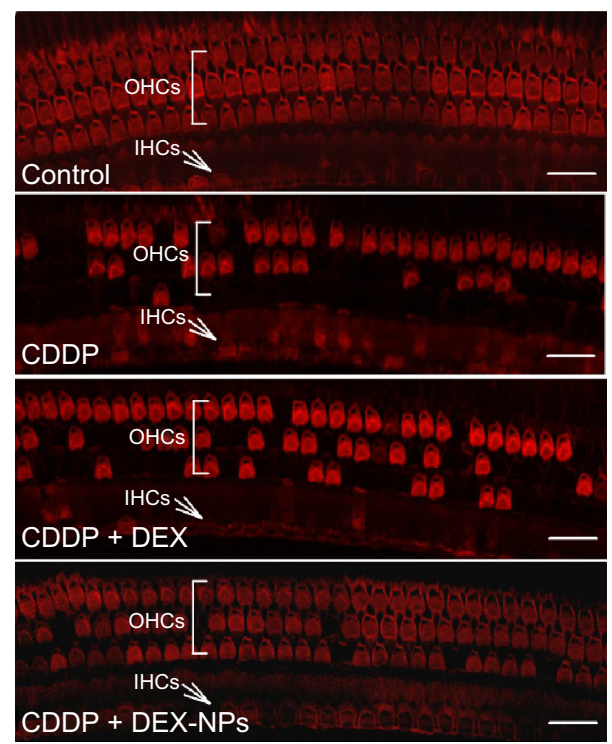

B
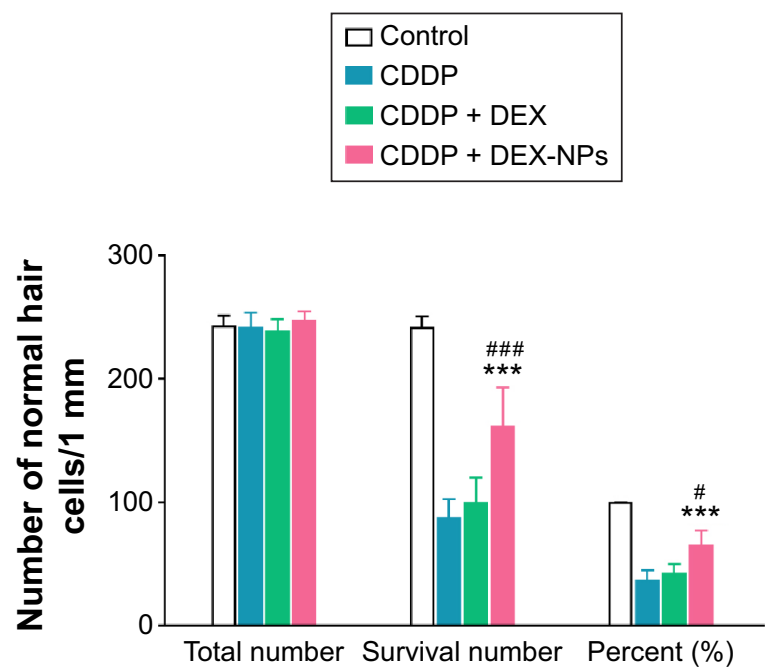

Figure 8 DEX-NPs alleviate cisplatin-induced damage on cochlear hair cells.

Notes: (A) Rhodamine phalloidin staining of the $60 \%$ portion from the apex of Corti. Severe damage to the OHCs was observed in the CDDP-and CDDP + DEX-treated animals, which were compared to CDDP + DEX-NPs. Scale bar, $40 \mu \mathrm{m}$. (B) Numbers and the percent survival of OHCs in I mm length about $60 \%$ from the apex of each cochlea $(n=6)$.

$$
\text { Total number }=\text { Survival number }+ \text { Absent number. }
$$

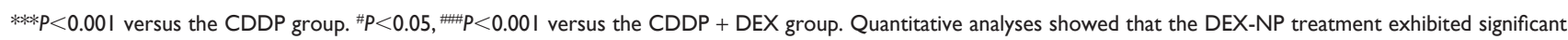
differences in the number and percentage of OHCs.

Abbreviations: OHCs, outer hair cells; IHCs, inner hair cells; CDDP, cis-diamminedichloroplatinumll; DEX, dexamethasone; DEX-NPs, dexamethasone-loaded polyethylene glycol-coated polylactic acid stealth nanoparticles; n, number.

\section{Inflammatory responses to DEX-NPs}

The middle ear was evaluated 3 days after the administration of DEX-NPs to assess histological changes in RWM (Figure 9). No inflammatory responses, including effusion or swelling of the mucosa, were observed in either the saline- (Figure 9A) or DEX-NP-treated ears (Figure 9B). However, a few inflammatory cells were present in the scala tympani near the RWM. The difference in the numbers of inflammatory cells between the DEX-NPs and saline groups was not significantly different.

\section{Discussion}

DEX, a glucocorticoid that acts as an otoprotectant, has been investigated in clinical trials in recent years. ${ }^{26}$ The potential mechanisms for otoprotection were proposed as inflammation modulation, ion homeostasis, and immune
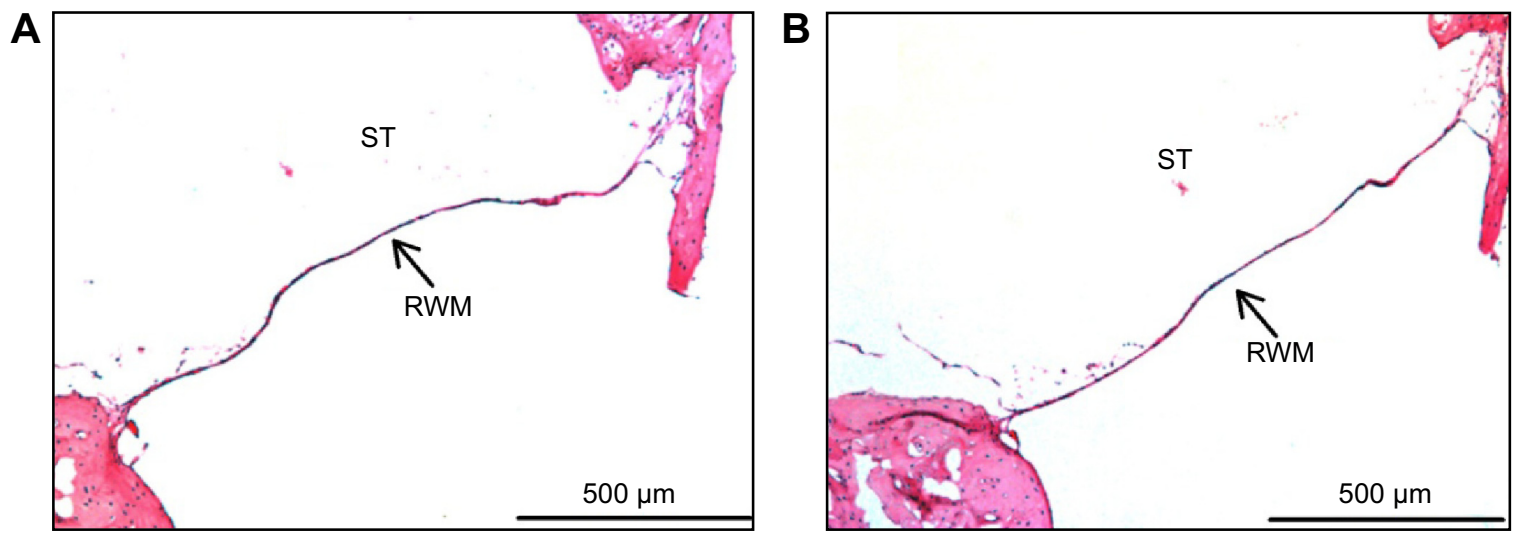

Figure 9 Hematoxylin and eosin-stained histological sections of a decalcified guinea pig middle-ear sample.

Notes: (A) RWM of the control ear with saline application. (B) RWM I day after the DEX-NPs administration. Blood is also visible in the scala tympani (n=3).

Abbreviations: RWM, round window membrane; ST, scala tympani; DEX-NPs, dexamethasone-loaded polyethylene glycol-coated polylactic acid stealth nanoparticles; n, number. 
suppression. ${ }^{27,28}$ DEX activity is mediated through the glucocorticoid receptor in the cochlea. ${ }^{28}$ Following systemic application, the amount of DEX available to the inner ear is limited after DEX molecules leave the circulation, cross the blood-cochlear barrier, and gain access to cells of the inner ear. ${ }^{29}$ Thus, a single injection of DEX at the clinically used dose may deliver very limited amounts of the drug into the cochlea. No protective effects of systemically administrated DEX against cisplatin-induced ototoxicity were observed in guinea pig models. ${ }^{10}$ These research findings suggested that DEX used by systemic administration in clinics may not be able to elicit a therapeutic effect in the cochlea.

Intratympanic drug delivery is recognized as a safe and simple drug administration route for inner ear disorders. This strategy has been widely used in the treatment of sudden sensorineural hearing loss and Meniere's disease. ${ }^{13}$ Protective effects of DEX through intratympanic delivery against cisplatin ototoxicity have been explored. However, the results to date are variable, depending on the doses, intensities, and frequencies of cisplatin injection or species of experimental animals. ${ }^{7,10,14-17}$ Among previous reports, two have employed a similar experimental setting to that of the present study, where guinea pigs were used as experimental animals and DEX was intratympanically administered. ${ }^{16,17}$ These two studies showed that the greatest otoprotection was achieved at the highest dose of DEX and reduced threshold shifts at $8 \mathrm{kHz}$ or $16 \mathrm{kHz}$. The DEX doses applied in these studies were higher than that used in the present study. The low dose of DEX in our study may not lead to sufficient exposure to the inner ear tissue for protection against cisplatin-induced ototoxicity.

NPs have attracted considerable interest for therapeutic application in ear disease owing to their sustained and controlled release properties. However, as yet, no published studies have reported the utility of NPs for delivering drugs to protect the ear against cisplatin-induced hearing loss. To our knowledge, this study is the first to demonstrate that DEX-NPs administered in a single dose via RWM exhibited a significant protective effect against cisplatin-induced hearing loss at both functional and histological levels by inducing an inflammatory reaction. Notably, an equivalent amount of free DEX applied onto the RWM failed to protect the inner ear against cisplatin-induced hearing loss.

The intracochlear concentration of DEX after RWM administration of free DEX reached a peak at 1 hour $(12,717.91 \pm 6,550.95 \mathrm{ng} / \mathrm{mL})$, but dropped to a very low level $(864.24 \pm 407.11 \mathrm{ng} / \mathrm{mL})$ after 6 hours. This indicates that the single administration of free DEX was quickly cleared from the cochlea, and that a more sustained exposure of DEX and a greater amount of DEX in the cochlea are needed to provide otoprotection. This may account for the poor effects of glucocorticoids in patients with cisplatin-induced ototoxicity after systemic application. $^{7}$

Particle size is one of the most important parameters that critically determines the fate of NPs in vitro and in vivo. ${ }^{30}$ It is noted that the sizes of DEX-NPs, as determined by DLS, TEM, and AFM, exhibited no significant differences. Strong fluorescence intensity of Coumarin 6-labeled NPs was found in the stria vascularis, and there was spiral prominence in the cochlear turns and cochlear modiolus 1 hour after RWM administration, whereas weak fluorescence of free Coumarin 6 was only detected in the cochlear modiolus after RWM administration. Coumarin 6 is used to study the distribution of NPs in targeted antitumor studies since it associates with NPs for a long time without dissociation. ${ }^{24}$ If Coumarin 6 had dissociated from NPs before it diffused into perilymph, we would expect less or equal amounts of Coumarin 6 in the inner ear as free Coumarin applied onto the RWM. However, we cannot exclude the possibility that NPs enhance the permeability of RWM. The RWM is a semipermeable membrane between the middle and inner ear, which allows the diffusion of particles $<3 \mu \mathrm{m}$ in diameter. ${ }^{29}$ The particle sizes of DEX-NPs and Coumarin 6-labeled NPs were less than $200 \mathrm{~nm}$ and could therefore easily penetrate the RWM into the perilymph of the scala tympani. A previous study has demonstrated that the cochlear fluid has an extremely slow flow rate; ${ }^{31}$ thus, the in vivo distribution of PEG-PLA NPs depends on the diffusion rates of NPs rather than the flow rate of the cochlear fluid.

The in vitro and in vivo release profiles of drug-loaded NPs represent the major parameters that influence the therapeutic effects. $^{32}$ The in vitro release rate of DEX from DEX-NPs followed this order: plasma $>$ AP $>$ PBS (Figure 1E). About $90 \%$ of DEX was released from the DEX-NPs in plasma medium in 24 hours, possibly due to the enzyme-catalyzed hydrolysis of the polymer matrix. In PBS and AP media, DEX displayed a biphasic release pattern; specifically, there was an initial fast release and a subsequent slower sustained release. In vivo, after the single-dose administration of DEXNPs, the DEX concentration in the perilymph of the cochlea reached a peak at 1 hour $(8,316.217 \pm 2,782.65 \mathrm{ng} / \mathrm{mL})$, lower than the DEX concentration of the free DEX administration at 1 hour $(12,717.91 \pm 6,550.95 \mathrm{ng} / \mathrm{mL})$ (Figure 4). Moreover, DEX was still present in the perilymph of the cochlea even after 48 hours of DEX-NPs administration. These results demonstrated that NPs are efficient to deliver DEX in a sustained manner to the cochlea. In addition, the 
application of Coumarin 6-labeled NPs revealed a wide and quick distribution in the organ of Corti, stria vascularis, and spiral ganglion cells throughout cochlea 1 hour after RWM administration, suggesting that NPs can accumulate in the hair cells and protect them against cisplatin-induced ototoxicity after being administrated.

Auditory function was further evaluated based on ABR thresholds and the results reflected the function of cochlear hair cells, spiral ganglion cells, and neurons in the auditory pathway. It has been documented that the use of cisplatin, like other ototoxic drugs, results in a loss of cochlear OHCs, starting in the base and progressing to the apex of the cochlea. ${ }^{33}$ Our study clearly demonstrated the protective effect of DEXNPs against cisplatin-induced damages to the $\mathrm{OHCs}$ at $4 \mathrm{kHz}$ and $8 \mathrm{kHz}$, but not at $16 \mathrm{kHz}$ and $32 \mathrm{kHz}$.

Decreased protection by DEX-NPs at the highest frequencies is expected, as the basal turn of the cochlea is the most susceptible to cisplatin ototoxicity. ${ }^{17}$ In general, destruction of OHCs due to drug toxicity progresses in a base-to-apex gradient. The cellular damage at basal turns of cochlea (high frequency) following a large, single dose of cisplatin may have been too severe to be prevented by RWM DEX-NPs. We found that the extensive loss of hair cells occurred along with a $25 \%$ mortality rate after the highest bolus dose of cisplatin at $12 \mathrm{mg} / \mathrm{kg}$ in our study. The cumulative and dose-related ototoxicity of cisplatin has been well documented. ${ }^{34}$ A lower dose of cisplatin is expected to trigger less damage to the cochlear sensory cells and hearing function, as observed in the current study. In clinical practice, cisplatin is usually administered in multiple lower doses over several days. This would result in less damage at the base of the cochlea, which might be further protected by the intratympanic application of DEX. ${ }^{15,16}$

\section{Conclusion}

In the present study, we developed a DEX-loaded NP drug delivery system for the treatment of ear disease. The resultant DEX-NPs were uniformly spherical in shape with a particle size of $130 \mathrm{~nm}$ (hydrodynamic diameter) and a zeta potential of $-20.16 \mathrm{mV}$. Coumarin 6-labeled NPs penetrated RWM rapidly and accumulated on the organ of Corti, stria vascularis, and ganglion cells in guinea pigs. The sustained release of DEX from DEX-NPs in vitro lasted for 24 days in PBS (pH 7.4), 5 days in AP, and 1 day in rat plasma. After the single-dose administration of DEX-NPs via RWM, the concentration of DEX in the cochlea of guinea pigs remained detectable for up to 48 hours. The sustained release property of the nanoparticulate system led to the prolonged retention of DEX, and to the significant attenuation of cisplatin-induced hearing loss in the cochlea, as examined at both functional and histological levels.

\section{Acknowledgments}

This work was supported by the Major Program of the National Natural Science Foundation of China (81330023), National Natural Science Foundation of China (81170924 and 81302709), Shanghai Committee of Science and Technology (14DZ2260300), Shanghai municipal foundation for young scientists (2012Y343), and the Xinhua Hospital Foundation (13YJ19).

\section{Disclosure}

The authors report no conflicts of interest in this work.

\section{References}

1. Gonçalves MS, Silveira AF, Teixeira AR, Hyppolito MA. Mechanisms of cisplatin ototoxicity: theoretical review. J Laryngol Otol. 2013; 127(6):536-541.

2. Schweitzer VG. Cisplatin-induced ototoxicity: the effect of pigmentation and inhibitory agents. Laryngoscope. 1993;103(4 Pt 2):1-52.

3. Benedetti Panici P, Greggi S, Scambia G, et al. Efficacy and toxicity of very high-dose cisplatin in advanced ovarian carcinoma: 4-year survival analysis and neurological follow-up. Int J Gynecol Cancer. 1993;3(1):44-53.

4. Eiamprapai P, Yamamoto N, Hiraumi H, et al. Effect of cisplatin on distortion product otoacoustic emissions in Japanese patients. Laryngoscope. 2012;122(6):1392-1396.

5. Li Y, Womer RB, Silber JH. Predicting cisplatin ototoxicity in children: the influence of age and the cumulative dose. Eur J Cancer. 2004; 40(16):2445-2451.

6. Mener DJ, Betz J, Genther DJ, Chen D, Lin FR. Hearing loss and depression in older adults. J Am Geriatr Soc. 2013;61(9):1627-1629.

7. Marshak T, Steiner M, Kaminer M, Levy L, Shupak A. Prevention of cisplatin-induced hearing loss by intratympanic dexamethasone: a randomized controlled study. Otolaryngol Head Neck Surg. 2014; 150(6):983-990.

8. Rybak LP, Whitworth CA. Ototoxicity: therapeutic opportunities. Drug Discov Today. 2005;10(19):1313-1321.

9. Van De Water TR, Abi Hachem RN, Dinh CT, et al. Conservation of hearing and protection of auditory hair cells against trauma-induced losses by local dexamethasone therapy: molecular and genetic mechanisms. Cochlear Implants Int. 2010;11 Suppl 1:42-55.

10. Waissbluth S, Salehi P, He X, Daniel SJ. Systemic dexamethasone for the prevention of cisplatin-induced ototoxicity. Eur Arch Otorhinolaryngol. 2013;270(5):1597-1605.

11. Herr I, Ucur E, Herzer K, et al. Glucocorticoid cotreatment induces apoptosis resistance toward cancer therapy in carcinomas. Cancer Res. 2003;63(12):3112-3120.

12. Chandrasekhar SS, Rubinstein RY, Kwartler JA, et al. Dexamethasone pharmacokinetics in the inner ear: comparison of route of administration and use of facilitating agents. Otolaryngol Head Neck Surg. 2000;122(4):521-528.

13. McCall AA, Swan EE, Borenstein JT, Sewell WF, Kujawa SG, McKenna MJ. Drug delivery for treatment of inner ear disease: current state of knowledge. Ear Hear. 2010;31(2):156-165. 
14. Calli C, Pinar E, Oncel S, Alper Bagriyanik H, Umut Sakarya E. Recovery of hearing in Cisplatin-induced ototoxicity in the Guinea pig with intratympanic dexamethasone. Indian J Otolaryngol Head Neck Surg. 2012;64(1):46-50.

15. Shafik AG, Elkabarity RH, Thabet MT, Soliman NB, Kalleny NK. Effect of intratympanic dexamethasone administration on cisplatin-induced ototoxicity in adult guinea pigs. Auris Nasus Larynx. 2013;40(1):51-60.

16. Murphy D, Daniel SJ. Intratympanic dexamethasone to prevent cisplatin ototoxicity: a guinea pig model. Otolaryngol Head Neck Surg. 2011; 145(3):452-457.

17. Hill GW, Morest DK, Parham K. Cisplatin-induced ototoxicity: effect of intratympanic dexamethasone injections. Otol Neurotol. 2008; 29(7):1005-1011.

18. Trune DR, Canlon B. Corticosteroid therapy for hearing and balance disorders. Anat Rec (Hoboken). 2012;295(11):1928-1943.

19. Rutt AL, Hawkshaw MJ, Sataloff RT. Incidence of tympanic membrane perforation after intratympanic steroid treatment through myringotomy tubes. Ear Nose Throat J. 2011;90(4):E21.

20. Slattery WH, Fisher LM, Iqbal Z, Friedman RA, Liu N. Intratympanic steroid injection for treatment of idiopathic sudden hearing loss. Otolaryngol Head Neck Surg. 2005;133(2):251-259.

21. Buckiová D, Ranjan S, Newman TA, et al. Minimally invasive drug delivery to the cochlea through application of nanoparticles to the round window membrane. Nanomedicine (Lond). 2012;7(9):1339-1354.

22. Yu DH, Lu Q, Xie J, Fang C, Chen HZ. Peptide-conjugated biodegradable nanoparticles as a carrier to target paclitaxel to tumor neovasculature. Biomaterials. 2010;31(8):2278-2292.

23. Bird PA, Begg EJ, Zhang M, Keast AT, Murray DP, Balkany TJ. Intratympanic versus intravenous delivery of methylprednisolone to cochlear perilymph. Otol Neurotol. 2007;28(8):1124-1130.

24. Gao C, Pan J, Lu W, Zhang M, Zhou L, Tian J. In-vitro evaluation of paclitaxel-loaded MPEG-PLGA nanoparticles on laryngeal cancer cells. Anticancer Drugs. 2009;20(9):807-814.
25. Müller M, von Hünerbein K, Hoidis S, Smolders JW. A physiological place-frequency map of the cochlea in the CBA/J mouse. Hear Res. 2005;202(1-2):63-73.

26. Rybak LP, Mukherjea D, Jajoo S, Ramkumar V. Cisplatin ototoxicity and protection: clinical and experimental studies. Tohoku J Exp Med. 2009;219(3):177-186.

27. Meltser I, Canlon B. Protecting the auditory system with glucocorticoids. Hear Res. 2011;281(1-2):47-55.

28. Horie RT, Sakamoto T, Nakagawa T, Ishihara T, Higaki M, Ito J. Stealth-nanoparticle strategy for enhancing the efficacy of steroids in mice with noise-induced hearing loss. Nanomedicine (Lond). 2010;5(9): 1331-1340.

29. Bowe SN, Jacob A. Round window perfusion dynamics: implications for intracochlear therapy. Curr Opin Otolaryngol Head Neck Surg. 2010; 18(5):377-385.

30. Alexis F, Pridgen E, Molnar LK, Farokhzad OC. Factors affecting the clearance and biodistribution of polymeric nanoparticles. Mol Pharm. 2008;5(4):505-515.

31. Ohyama K, Salt AN, Thalmann R. Volume flow rate of perilymph in the guinea-pig cochlea. Hear Res. 1988;35(2-3):119-129.

32. Feng L, Wu H, Ma P, Mumper RJ, Benhabbour SR. Development and optimization of oil-filled lipid nanoparticles containing docetaxel conjugates designed to control the drug release rate in vitro and in vivo. Int J Nanomedicine. 2011;6:2545-2556.

33. García-Berrocal JR, Nevado J, Ramírez-Camacho R, et al. The anticancer drug cisplatin induces an intrinsic apoptotic pathway inside the inner ear. Br J Pharmacol. 2007;152(7):1012-1020.

34. Bokemeyer C, Berger CC, Hartmann JT, et al. Analysis of risk factors for cisplatin-induced ototoxicity in patients with testicular cancer. Br J Cancer. 1998;77(8):1355-1362.
International Journal of Nanomedicine

\section{Publish your work in this journal}

The International Journal of Nanomedicine is an international, peerreviewed journal focusing on the application of nanotechnology in diagnostics, therapeutics, and drug delivery systems throughout the biomedical field. This journal is indexed on PubMed Central, MedLine, CAS, SciSearch $®$, Current Contents $\AA /$ Clinical Medicine,

\section{Dovepress}

Journal Citation Reports/Science Edition, EMBase, Scopus and the Elsevier Bibliographic databases. The manuscript management system is completely online and includes a very quick and fair peer-review system, which is all easy to use. Visit http://www.dovepress.com/ testimonials.php to read real quotes from published authors. 